\section{Protocol deviations revisited}

\section{To the editor:}

In the June 2008 "Protocol Review" column, there was a discussion about whether performing fewer procedures than originally planned constitutes a significant deviation that ought to be reported to OLAW ${ }^{1}$. The members of the American Physiological Society (APS) Animal Care and Experimentation Committee take exception to the view expressed by several commentators that reducing the number of procedures performed in a protocol necessitates IACUC review.

This view seems to have originated in question 9 of the Frequently Asked Questions (FAQs) section of the OLAW website (http://grants.nih.gov/grants/olaw/faqs.htm\#proto_9), which presents a discussion of "significant" protocol changes that require IACUC review. The response to question 9 is based on a 1995 article in Lab Animal, wherein Potkay et al. provided examples of things that might constitute "significant changes" to an approved research protocol ${ }^{2}$. The examples included changing the objectives of a study, changing the approximate numbers of animals used or withholding analgesics. The authors also mentioned "additional factors" for the IACUC to consider, including "changes in the duration, frequency, or number of procedures performed on an animal." In the FAQ, a change seems to have occurred so that the duration, frequency and number of procedures are listed as examples of significant changes rather than as additional factors.

There is no dispute that increases in the duration, frequency or number of procedures constitute significant changes to a protocol. However, the converse notion that decreases must be reviewed for their significance does not make sense. Although IACUC review is needed in certain cases where fewer procedures than planned are performed-for example, when procedures intended to promote animal welfare are eliminated-review should not be required in all cases. Experimental work is dynamic, and modifications are often needed. At a certain point, IACUCs have to rely on the professional judgment of Principal Investigators (PIs) to produce scientifically valid results while maintaining the integrity of the work as approved by the IACUC. Expecting IACUCs to screen each change for its significance would place these committees in the position of micromanaging every protocol. This would have enormous implications for the workload of both IACUCs and PIs.

This approach would also make it more difficult for scientists to refine procedures, a practice that benefits both the quality of the science and the welfare of animals. Forcing scientists to seek permission every time they want to eliminate a procedure that proves to be unnecessary or counterproductive would encumber a simple yet far-reaching means by which scientists could otherwise incorporate the $3 \mathrm{Rs}$ into daily practice.

For these reasons, the APS Animal Care and Experimentation Committee believes that it is incumbent upon OLAW to clarify whether or not it intended for decreases in the number of procedures to be considered by default to be significant changes to a protocol.

\section{Alice Ra'anan}

Director of Government Relations and Science Policy

The American Physiological Society

9650 Rockville Pike

Bethesda, MD 20814-3991

1. Silverman, J. et al. Which protocol deviations require preapproval? Lab Anim. (NY) 37, 345-346 (2008).

2. Potkay, S., Garnett, N., Miller, J.G., Pond, C.L. \& Doyle, D.J. Frequently asked questions about the Public Health Service Policy on humane care and use of laboratory animals. Lab Anim. (NY) 24, 24-26 (1995).

\section{OLAW replies:}

In response to the issues raised by the American Physiological Society's Animal Care and Experimentation Committee, OLAW offers the following clarification.

The Public Health Service Policy on Humane Care and Use of Laboratory Animals ${ }^{1}$ requires the IACUC to "review and approve, require modifications in (to secure approval) or withhold approval of proposed significant changes regarding the use of animals in ongoing activities." OLAW's FAQs (http:/grants.nih.gov/grants/ olaw/faqs.htm) provide OLAW's responses to general questions from institutions and IACUCs as they implement the PHS Policy. Information in the FAQs is updated frequently.

Owing to the great diversity of research performed at Assured institutions, the OLAW FAQ on the subject of protocol changes that require IACUC review provides a list of examples of the types of changes that OLAW considers to be significant and explains that the IACUC has "discretion to define what it considers a significant change, or to establish a mechanism for determining significance on a case-by-case basis."

OLAW agrees with the APS Animal Care and Experimentation Committee's statement that "IACUC review is needed in certain cases where fewer procedures than planned are performed." It is incumbent on PIs, when they have doubts about a change in an animal research activity, to communicate with their IACUC. As stated in the FAQ, it is also critical that the IACUC clearly define and communicate to investigators what it considers to be a significant change, or its mechanism for determining significance.

OLAW welcomes comments on any of its guidance. Comments may be submitted to OLAW by email (olaw@od.nih.gov). All relevant comments will be considered in OLAW decisions on timing and content of revisions to guidance documents or development of new guidance documents.

\section{Patricia Brown, VMD, MS, DACLAM}

Director OLAW, OER, OD, NIH, HHS

email:olaw@od.nih.gov

1. Public Health Service. Policy on Humane Care and Use of Laboratory Animals IV.B.7 (US Department of Health and Human Services, Washington, DC, 1986; amended 2002). 